\title{
Simulation and Experimental Verification of a Bimorph Beam Piezoelectric Energy Harvesting Devices
}

\author{
Siyang Zhang, Yexin Chen \\ Department of Mechanical and Electrical Engineering, Zhixing College of Hubei University, Wuhan \\ 430011, China
}

Keywords: Piezoelectric; bimorph; energy harvester; natural frequency.

\begin{abstract}
A bimorph beam piezoelectric energy harvesting device is investigated in this paper. The expression of voltage and power outputs are obtained by mathematical analysis in both series and multiple models. The simulation for the piezoelectric energy harvester is based on finite element method, and the characteristic relation of the physical dimensions, mass, spring stiffness coefficient and natural frequency is obtained. Finally, experiments are conducted to verify and study the influence on voltage output from excited frequency.
\end{abstract}

\section{Introduction}

There are many types of energy harvester, such as Bimorph beam structure [1]. Bimorph beam structure is widely used, as it has many advantages including: simple structure, good machinability [2], low cost, etc. Many researches are conducted on increasing the bandwidth of bimorph beam piezoelectric energy harvesting device [3], and most of them focus on the structure optimization.

\section{Model of a Bimorph Beam Piezoelectric Energy Harvesting Device}

Research is based on a mechanism which includes mass block, piezoelectric bimorph beam, and excited frequency, as shown in Fig.1. The width of rectangular of upper layer and lower layer are the same and the shapes are symmetrical. From top to bottom are upper piezoelectric layer (PZT material), $\mathrm{Cu}$ base layer (support layer), and lower piezoelectric layer (PZT material). Piezoelectric layers and base layer are combined by binder (epoxy resin), and surfaces of upper and lower piezoelectric layer are covered by Electrode layer $(\mathrm{Cu}$ film).

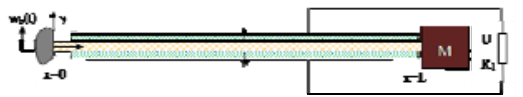

(a)Series circuit

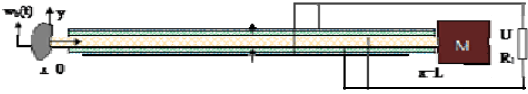

(b)Parallel circuit

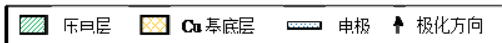

Fig 1. Piezoelectric bimorph beam

The "Spring-Mas-Damping-Piezoelectric" model are proposed to analysis the influence factors of voltage and power output.

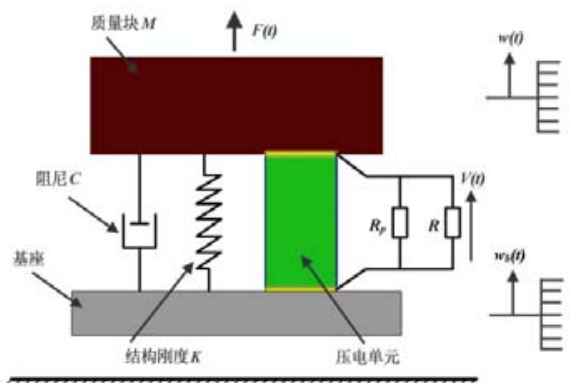

Fig 2. The "Spring-Mas-Damping-Piezoelectric" model 
The model consists of the mechanical components and piezoelectric components, $\mathrm{M}$ is the mass of block, $\mathrm{C}$ is the damping coefficient, and $\mathrm{K}$ represents the stiffness of spring. $\mathrm{RP}$ is the internal resistance. Parallel circuit consists of RP and another load which is $\mathrm{R}$. web (t) represents the excitation input and $w(t)$ is the displacement of the freedom end of a cantilever beam.

Assuming the excitation input is web ( $\mathrm{t}$ ), based on equilibrium equation, the model can be expressed as follows:

$$
M \ddot{\omega}(t)+b_{m} \dot{\omega}(t)+K \omega(t)+\chi_{b c} V(t)=-M \ddot{\omega}_{b}(t)
$$

Series circuit and Parallel circuit are two different circuits in energy harvesting device, and will be discussed separately as follows:

$d_{31}$ is Piezoelectric strain constant, $c_{11}^{E}$ is the stiffness coefficient of spring $e_{31}=d_{31} c_{11}^{E}, t_{p}$ is the thickness of Piezoelectric layer, $A_{p e}$ is the effective area of Piezoelectric layer, $C_{e}$ is the internal current, $\zeta_{m}$ is the damping ratio of the system, $\omega_{n}$ is the inherent frequency of the system.

The voltage output $|V|$ and power $P$ output of the piezoelectric bimorph beam (Series circuit) are:

$$
\left\{\begin{array}{l}
|V|=\frac{\omega \frac{2 c_{11}^{E} d_{31} t_{\rho} \kappa_{b} A_{n}}{\varepsilon}}{\sqrt{\left[\frac{2 \omega_{n}^{2}}{R C_{e}}-\omega^{2}\left(6 \zeta_{m} \omega_{n}+\frac{2}{R C_{e}}\right)+j \omega\left(\omega_{n}^{2}\left(3+2 k^{2}\right)+\frac{4 \zeta_{m} \omega_{n}}{R C_{e}}-3 \omega^{2}\right)\right]^{2}}} \\
P=\frac{|V|^{2}}{2 R}
\end{array}\right.
$$

The maximum output can be obtained when $\omega=\omega_{n}$, and the results are as follows:

$$
\begin{gathered}
|V|_{\omega_{n}}=\frac{\omega_{n} \frac{c_{11}^{E} d_{31} t_{p} \kappa_{b} A_{i n}}{2 \varepsilon}}{\sqrt{\left[-\omega_{n}^{3} 2 \zeta_{m}+j \omega_{n}\left(\frac{1}{2} k^{2} \omega_{n}^{2}+\frac{\zeta_{m} \omega_{n}}{2 R C_{e}}\right]^{2}\right.}} \\
P_{\omega_{n}}=\frac{\frac{1}{4}\left(\omega_{n} c_{11}^{E} d_{31} t_{p} \kappa_{b} A_{i n}\right)^{2}}{R \varepsilon^{2}\left[-\omega_{n}^{3} 2 \zeta_{m}+j \omega_{n}\left(\frac{1}{2} k^{2} \omega_{n}^{2}+\frac{\zeta_{m} \omega_{n}}{2 R C_{e}}\right)\right]^{2}}
\end{gathered}
$$

\section{Structure Optimization Based on Finite Element Analysis}

\subsection{Piezoelectric Bimorph Beam Model.}

Piezoelectric bimorph beam model is shown as figure 3.

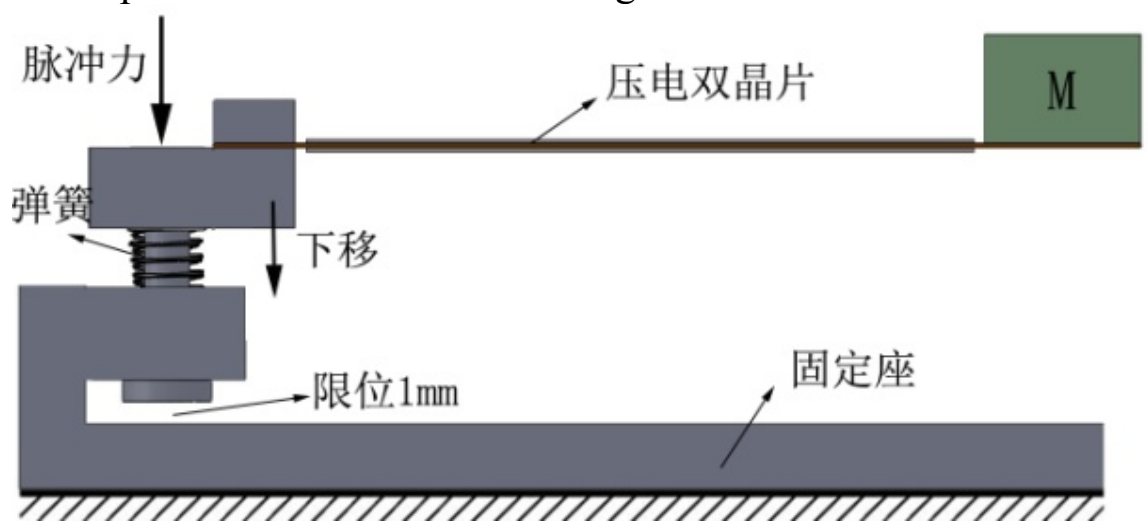

Fig 3. Piezoelectric bimorph beam model

There is a block with mass $\mathrm{M}$ at the free end of the cantilever beam. The fixed end of the cantilever beam is connected by a spring. In terms of the base layer, the length is $45 \mathrm{~mm}$, width is $15 \mathrm{~mm}$, and thickness is $0.2 \mathrm{~mm}$. Regarding the piezoelectric layer, the length is $35 \mathrm{~mm}$, width is $15 \mathrm{~mm}$, and thickness is $0.2 \mathrm{~mm}$. The three dimensions of the mass block are $10 \mathrm{~mm}, 15 \mathrm{~mm}, 5 \mathrm{~mm}$, respectively. Regarding the spring, its stiffness is $48 \mathrm{~N} / \mathrm{mm}$, and its length is $5 \mathrm{~mm}$. The base material is Brass, the piezoelectric material is PZT-5H, and the block material is tungsten alloy.

The piezoelectric beam model in ANSYS before the calculation is shown in figure 4. 


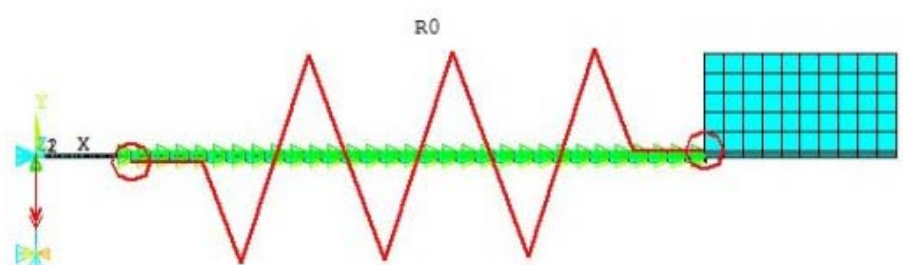

Fig 4. The piezoelectric beam model in ANSYS before the calculation

\subsection{Simulation and Optimization.}

The modal analysis of the piezoelectric beam is shown in Table 1.

Table 1 . The modal analysis of the piezoelectric beam

\begin{tabular}{ccccc}
\hline order & One order & Two order & Three order & Four order \\
\hline resonant frequency $/ \mathrm{Hz}$ & 11.228 & 200.27 & 994.61 & 1302.6 \\
\hline
\end{tabular}

The harmonic response analysis result around its first order nature frequency is shown in Figure 5.

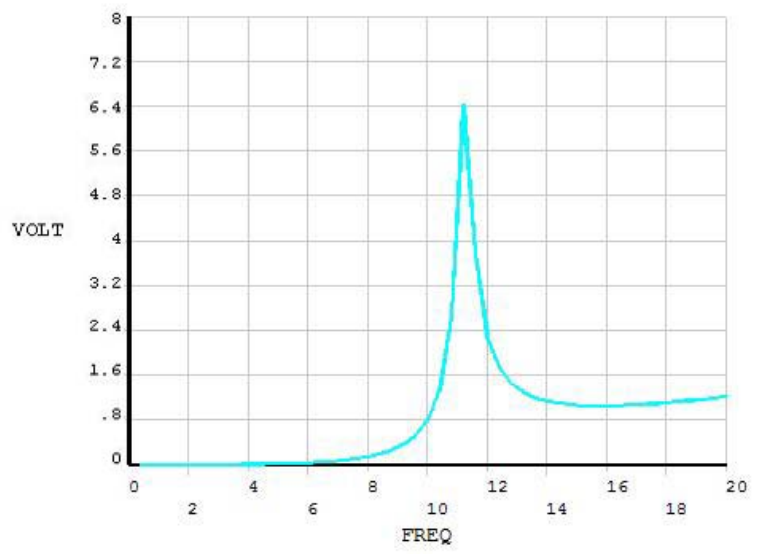

Fig 5. The harmonic response analysis result

From the figure 5 , it can be easily founded that the maximal voltage output is $6.4 \mathrm{~V}$, and the bandwidth is around $2 \mathrm{~Hz}$.

\section{Experiment Verification}

The physic model of piezoelectric bimorph cantilever beam is built to verify the simulation, which can be seen in Figure 6. The experiment sample consists of: (1) piezoelectric ceramics PZT-5H $(50 \mathrm{~mm} \times 50 \mathrm{~mm} \times 0.2 \mathrm{~mm})$, (2) block with mass of $0.015 \mathrm{~kg}$, (3) fixture of the cantilever beam, (4) connection wire, (5) laser reflection surface, (6) base. The parallel connection is selected here. The fixed end of the cantilever beam and the electrode are connected to the fixture. The input is given to the beam through the fixture.
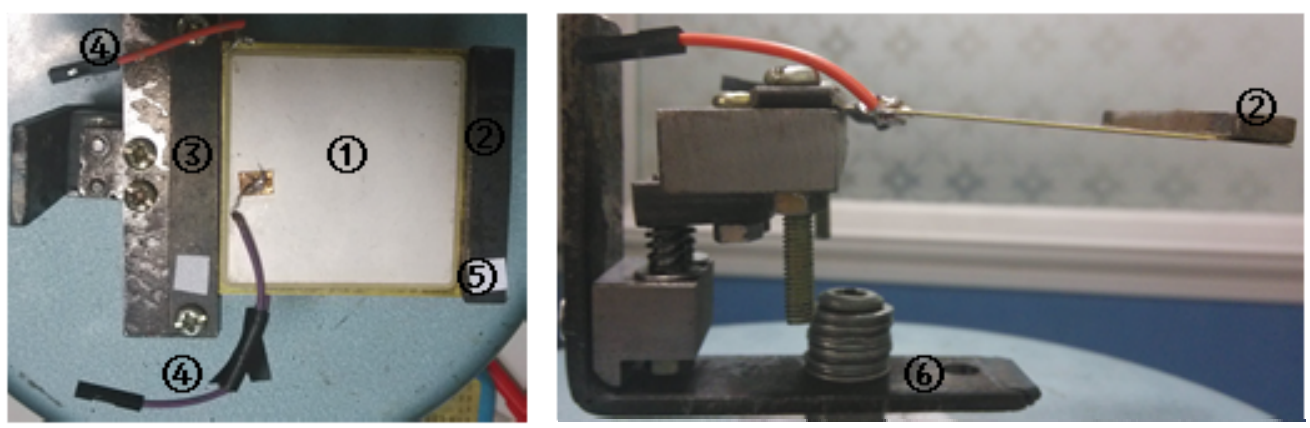

Fig 6. The physic model of piezoelectric bimorph cantilever beam 


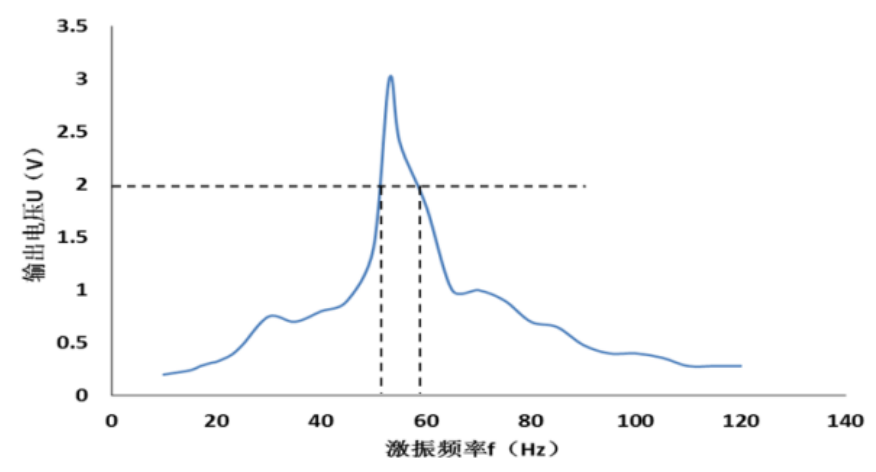

Fig 7. Output voltage and excitation frequency

The experiment results are in accordance with the simulation results. The relation between output voltage and excitation frequency is built as figure 7 . The maximum output voltage is $5 \mathrm{~V}$, which is at its first order nature frequency $55 \mathrm{~Hz}$. As the excitation frequency increase, the output voltage increases gradually at the beginning and rise drastically after $40 \mathrm{~Hz}$, on the other hand, the voltage decreases rapidly at the beginning and decrease gradually when the excitation frequency is higher than $55 \mathrm{~Hz}$. Taking $2 \mathrm{~V}$ as effective voltage, the effective bandwidth of the system is $7 \mathrm{~Hz}$.

\section{Conclusion}

The mathematical model of series circuit and parallel circuit are built, the expression of voltage and power outputs are obtained by mathematical analysis. According to the Simulation, the relation between output and the influence factors such as piezoelectric bimorph beam dimensions, mass of the block, spring stiffness, are investigated. Finally, the physic model of piezoelectric bimorph cantilever beam is built to analysis how excitation frequency affect voltage output.

\section{References}

[1]. S.J. Roundy, Energy Scavenging for Wireless Sensor Nodes with a Focus on Vibration to Electrical Conversion[D]. Univ. of California, Berkeley,2003.

[2]. Jong S J, Kim M S, Song J S, Two-layered piezoelectric bender device for micro-power generator[J]. Sensors and Actuators, 2008, 148(1): 158-167.

[3]. Challah V R, Prasad M G, Shi Y, et al. A vibration energy harvesting device with bidirectional resonance frequency tenability [J]. Smart Materials and Structures, 2008, 17:1-10. 